\title{
SOFT SPIN PHYSICS AT JEFFERSON LABORATORY
}

\author{
R. DE VITA \\ Istituto Nazionale di Fisica Nucleare, \\ via Dodecaneso 33, 16146 Genova, Italy \\ E-mail: devita@ge.infn.it
}

\begin{abstract}
An extensive experimental program to study spin physics at low and moderate four-momentum transfer, $Q^{2}$, is in progress at Jefferson Lab. In this regime, soft processes as resonance excitation and higher twist contribution play a dominant role and the measurement of spin observables is a fundamental tool to understand such phenomena and identify the relevant degrees of freedom. In these proceedings I will describe the ongoing experimental program and I will discuss preliminary and final results.
\end{abstract}

\section{Physics Motivation}

In the last three decades extensive studies of the spin properties of the nucleon have been carried out at large momentum transfer $Q^{2}$. Measurements of the spin-dependent structure functions $g_{1}$ and $g_{2}$ have been performed in several facilities as SLAC, CERN, and DESY ${ }^{1}$ in the DIS region, providing first information on the spin-dependent parton distribution functions.

As $Q^{2}$ decreases, non-perturbative effects as resonance excitations start to play a dominant role and the connection between the nucleon properties and its elementary constituents, i.e. quarks and gluons, becomes highly non-trivial. In particular at low $Q^{2}$, a better description of the nucleon can be obtained in terms of hadronic degrees of freedom.

Understanding the transition region between hadronic and partonic degrees of freedom is a key issue in strong interaction physics and spin observables are a fundamental tool to improve our knowledge of these phenomena.

A broad program to study the nucleon spin structure in the soft regime is in progress at Jefferson Lab involving the three experimental Halls of the facility with the goal of mapping this kinematic region. This ongoing program includes measurements of the nucleon spin structure functions in the resonance region and of their integrals, measurements of nucleon spin polarizabilities at low $Q^{2}$, test of duality of spin structure functions, study 
of higher twist contributions, and the study of polarization observables in exclusive and semi-inclusive meson production. In the next sections I will describe the experimental setup used in such measurements and I will then discuss a selection of the already available results focusing on the low $Q^{2}$ domain. Other information on the Jefferson Lab spin physics program and results can be found in Ref. 2, 3, 4, 5 .

\section{Experimental Setup}

The core of Jefferson Lab is the CEBAF accelerator (Continuous Electron Beam Accelerator Facility). This consists of two superconducting linacs connected by recirculation arcs. The superconductive cavities operate at a frequency of $1.497 \mathrm{GHz}$, resulting in a $2.0 \mathrm{~ns}$ duty cycle beam for the three experimental halls (Hall A, B, and C). The electron beam is produced by illuminating a strained GaAs cathode with a gain switched laser diode, operating at $780 \mathrm{~nm}$. The resulting electron polarization has typical values of $70-80 \%$. The injected electrons can circulate up to five times reaching a maximum energy of $5.75 \mathrm{GeV}$.

The three experimental halls are equipped with complementary instrumentation.

The Hall A spin physics experiments $6,7,8,9,10,11$ have been performed using a polarized Helium-3 gas target as an effective polarized neutron target. ${ }^{12}$ The ${ }^{3} \mathrm{He}$ nuclei are polarized through the spin exchange with optically pumped Rubidium atoms reaching typical polarization of $35 \%$. The scattered electrons are detected by two identical high resolution spectrometers. The orientation of the spectrometers with respect to the electron beam line was varied to cover a wide range of $Q^{2}$ and invariant mass $W$.

Hall B is equipped with the CEBAF Large Acceptance Spectrometer (CLAS) ${ }^{13}$ Exploiting the large acceptance of the detector, inclusive, semiinclusive, and exclusive measurements ${ }^{14,15,16,17}$ were performed simultaneously using a solid state $\mathrm{NH}_{3} / \mathrm{ND}_{3}$ target, polarized longitudinally through Dynamic Nuclear Polarization. ${ }^{18}$

Finally Hall C is instrumented with two spectrometers with different resolution and acceptance. Measurement of inclusive asymmetries and structure functions on proton and deuteron ${ }^{19}$ were performed using a $\mathrm{NH}_{3} / \mathrm{ND}_{3}$ target. ${ }^{20}$ The target polarization can be both parallel and perpendicular to the electron beam direction, allowing the full separation of the nucleon structure functions $g_{1}$ and $g_{2}$.

The complementarity of the experimental setups of the three Halls has 

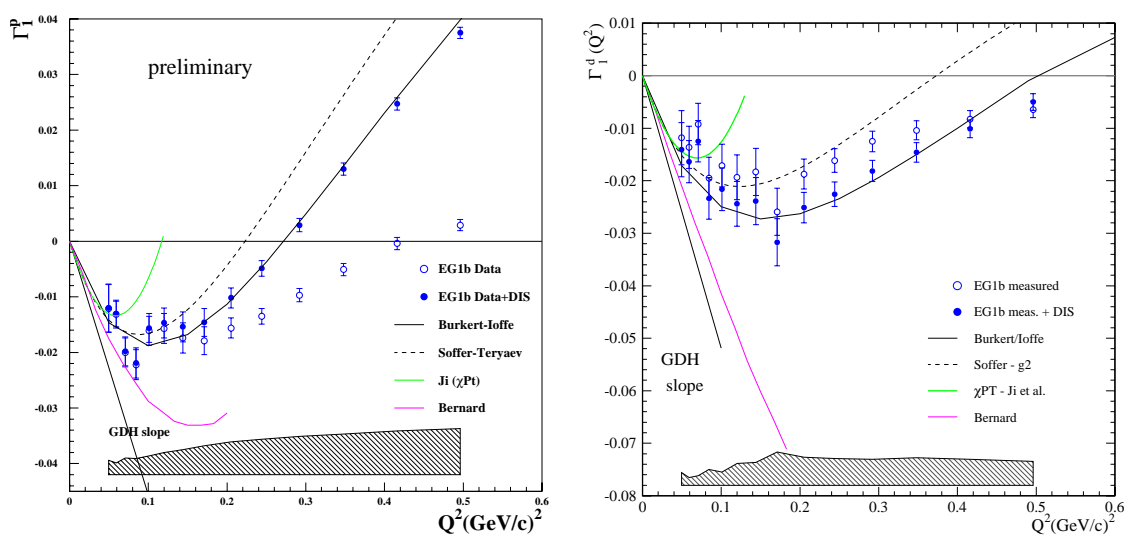

Figure 1. Preliminary results on $\Gamma_{1}\left(Q^{2}\right)$ for the proton (left) and for the deuteron (right) obtained by CLAS. The open circles are the CLAS data integrated over the measured region, while the full circles include the DIS contribution. The shaded band shows the systematic error associated with the measurement. At low $Q^{2}$ the slope given by the GDH sum rule and chiral perturbation theory calculation from Ref. 25 and 26 are indicated. The dashed and dotted lines are model predictions of Ref. 27 and 28.

allowed the completion of a broad and extensive physics program and has provided the possibility of performing important checks of possible systematic effects in the extraction of the observables of interest.

\section{Sum Rules and Integrals of Spin Structure Functions}

One of the primary goals of the Jefferson Lab spin physics program is the measurement of the spin structure function $g_{1}\left(x, Q^{2}\right)$ and $g_{2}\left(x, Q^{2}\right)$ on the proton and neutron, and extraction of their integrals $\Gamma_{1}\left(Q^{2}\right)=$ $\int g_{1}\left(x, Q^{2}\right) d x$ and $\Gamma_{2}\left(Q^{2}\right)=\int g_{2}\left(x, Q^{2}\right) d x$. These are connected to important sum rules as the Bjorken sum rule which relates the difference $\left(\Gamma_{1}^{p}-\Gamma_{1}^{n}\right)$ to the axial form factor of the nucleon in the infinite $Q^{2}$ limit ${ }^{21}$, and the Burkhardt-Cottingham sum rule which predicts $\Gamma_{2}\left(Q^{2}\right)$ to be zero. ${ }^{22}$

The $\Gamma_{1}^{p}$ and $\Gamma_{1}^{n}$ integrals have been measured at large $Q^{2}$ at SLAC, CERN, and DESY, and have been found to be respectively positive and negative, consistently with what expected based on the Bjorken sum rule and pQCD calculations. On the contrary both integrals are expected to be negative at very low $Q^{2}$ and to approach zero for $Q^{2} \rightarrow 0$ with a negative slope based on the relation between the $\Gamma_{1}$ integral and the Gerasimov-DrellHearn sum rule. ${ }^{23,24}$ This sum rule relates the difference of the helicity- 
dependent total photoabsorption cross sections to the anomalous magnetic moment of the the nucleon and is based on very general assumptions, such as gauge invariance, causality, and analyticity. Because of this constraint, the $\Gamma_{1}$ integral in particular for the proton must undergo to a dramatic change as $Q^{2}$ varies from the photon point to the perturbative domain.

The $\Gamma_{1}$ integrals for proton and deuteron have been measured in Hall B with CLAS, while $\Gamma_{1}^{n}$ has been extracted from the ${ }^{3} \mathrm{He}$ measurement performed in Hall A.

Figure 1 shows the CLAS measurements on proton and deuteron up to $Q^{2}=0.5 \mathrm{GeV}^{2}$. The open circles shows the integral of $g_{1}$ over the measured region, while the full circles include the extrapolation at small $x$ evaluated using a parameterization of the world data. The error bars represent the statistical error while the shaded band shows the systematic uncertainty. At low $Q^{2}$ the slope given by the GDH sum rule is shown as well as the chiral perturbation theory predictions of Bernard ${ }^{25}$ and $\mathrm{Ji}^{26}$. The dashed and dotted line are model predictions of Ref. 27 and 28. The measured integrals are consistent with the expected behavior both for the proton and deuteron showing the change in slope for $Q^{2} \sim 0.1-0.2 \mathrm{GeV}^{2}$. The comparison with $\chi \mathrm{PT}$ predictions shows good agreement only at the lower $Q^{2}$ while significant discrepancies are found above.

Figure 2 shows the $\Gamma_{1}$ (top) and $\Gamma_{2}$ (middle) integrals for the neutron, measured in Hall A. ${ }^{29}$ The $\Gamma_{1}$ integral measured over the resonance region is shown by the squares, while the full circles include the extrapolation in the unmeasured region based on the parameterization by Bianchi and Thomas. ${ }^{31}$ The size of the points represents the statistical error while the shaded band shows the systematic uncertainty. Previous measurements from SLAC and HERMES and different theoretical predictions from Ref. 26, 25, 27, 28, 32 are shown. The new Hall A data agree fairly well with the previous measurements with much improved accuracy, extending the coverage at very low $Q^{2}$ where $\chi \mathrm{PT}$ predictions are available. The Burkhardt Cottingham sum rule on the neutron has been tested integrating the structure function $g_{2}\left(x, Q^{2}\right)$. The results shown in the middle panel of figure 2 seem to indicate that the sum rule is verified within the experimental uncertainty .

The Hall A and Hall B measurements of $\Gamma_{1}$ on proton, deuteron, and neutron have been combined to extract the Bjorken integral. ${ }^{30}$ Details of this analysis and results can be found in Ref. 3 . 


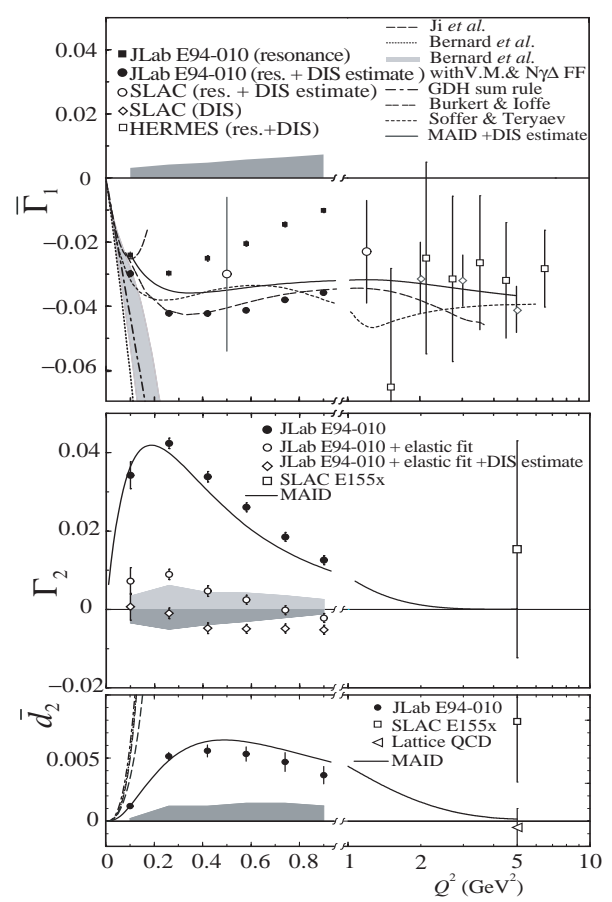

Figure 2. Top: $\Gamma_{1}$ integral for the neutron. The squares show the integral over the measured region while the full circles includes the extrapolation at small $x$. The previous SLAC and Hermes measurements are shown for comparison. Middle: $\Gamma_{2}$ integral for the neutron. The full circles show the measured integral while the open circle include the elastic contribution. The positive shaded band shows the experimental uncertainties, while the negative band shows the effect of the extrapolation at small $x$. Bottom: $d_{2}\left(Q^{2}\right)$ integral for the neutron. The smooth curve shows the prediction of the MAID model. At $5 \mathrm{GeV}^{2}$ the SLAC data give a positive value while lattice QCD calculations give a small and negative value.

\section{Duality in Spin Structure Functions}

A dual nature of the nucleon structure functions was first observed in the unpolarized sector by Boom and Gilman ${ }^{33}$ who noticed that the nucleon structure function $\nu W\left(x, Q^{2}\right)$ measured in the resonance region at low $Q^{2}$ was equivalent to the structure function $F_{2}(x)$ measured in the DIS regime when averaged over the same range of the scaling variable $\xi=2 x /(1+$ $\left.\sqrt{1+4 M^{2} x^{2} / Q^{2}}\right)$. Duality for unpolarized structure functions has been found to hold at the $10 \%$ level down to $Q^{2} \sim 1 \mathrm{GeV}^{2}$.

In the polarized sector, the situation is more complicated due to the resonance contribution which can bring the structure functions to have 


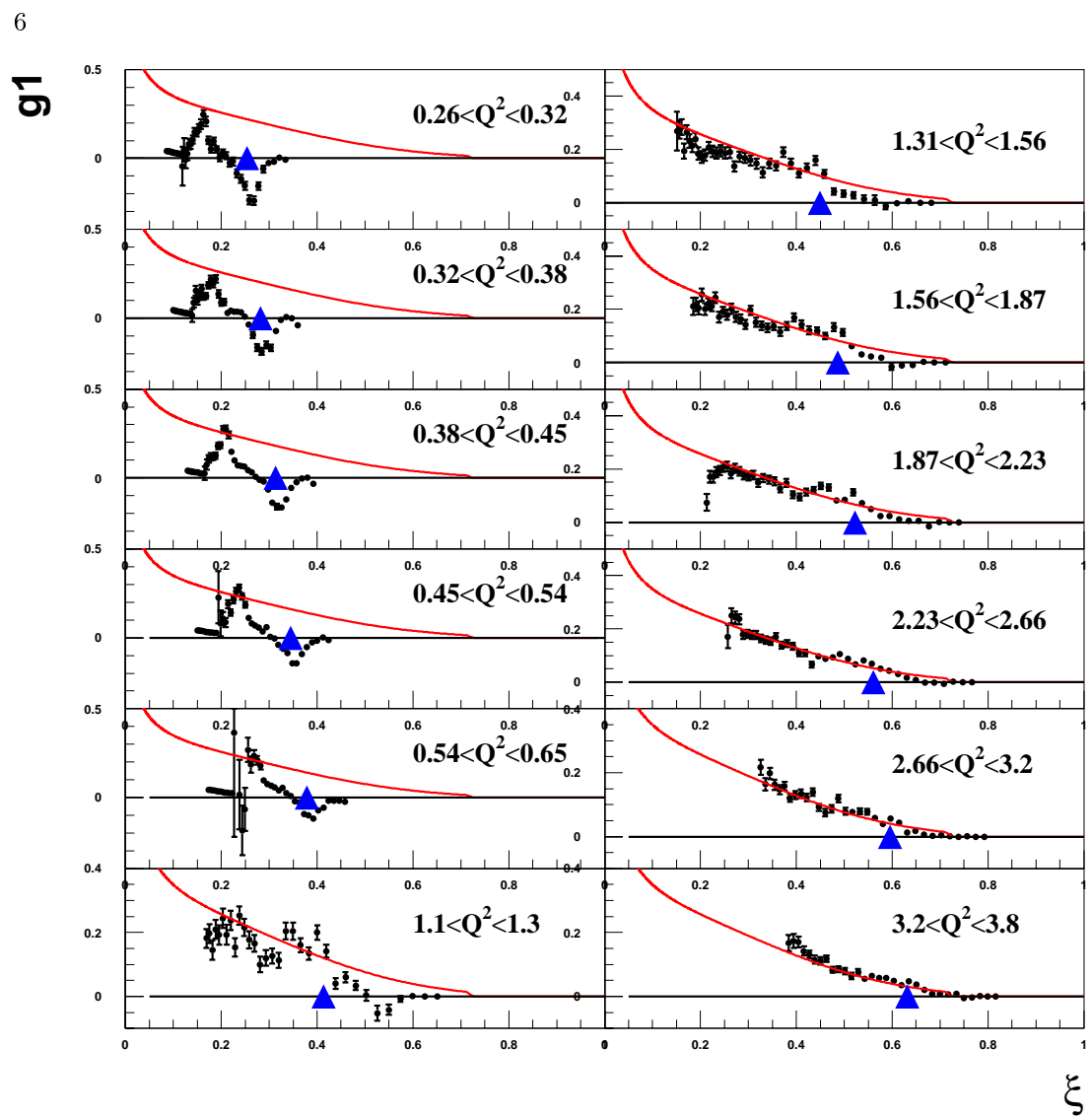

Figure 3. $g 1$ structure function on the proton from CLAS. The line shows a parameterization of the world data evolved at $Q^{2}=10 \mathrm{GeV}^{2}$. The triangle indicates the position of the $\Delta(1232)$ resonance.

negative values as shown in Figure. 3. A first test of duality of the proton structure function $g_{1}$ was performed by the HERMES Collaboration comparing the integral of $g_{1}$ over a specific $x$ range in the resonance region, $\Gamma_{1}^{R E S}\left(Q^{2}\right)=\int_{x 1}^{x 2} g_{1}\left(x, Q^{2}\right) d x$, with that over an equivalent region of $x$ in the DIS regime, $\Gamma_{1}^{D I S}=\int_{x 1}^{x 2} g_{1}(x) d x$. The HERMES Collaboration found $\Gamma_{1}^{R E S}\left(Q^{2}\right) / \Gamma_{1}^{D I S}$ to be consistent with unity at the $20 \%$ level down to the minimum $Q^{2}$ of $\sim 1.5 \mathrm{GeV}^{2} .{ }^{34}$ A similar analysis has been performed using the CLAS data on the proton and deuteron structure functions. Figure 4 shows the $\Gamma_{1}^{R E S}\left(Q^{2}\right) / \Gamma_{1}^{D I S}$ ratio for the proton (top) and deuteron (bottom) as a function of $Q^{2}$. The CLAS preliminary results are in general 


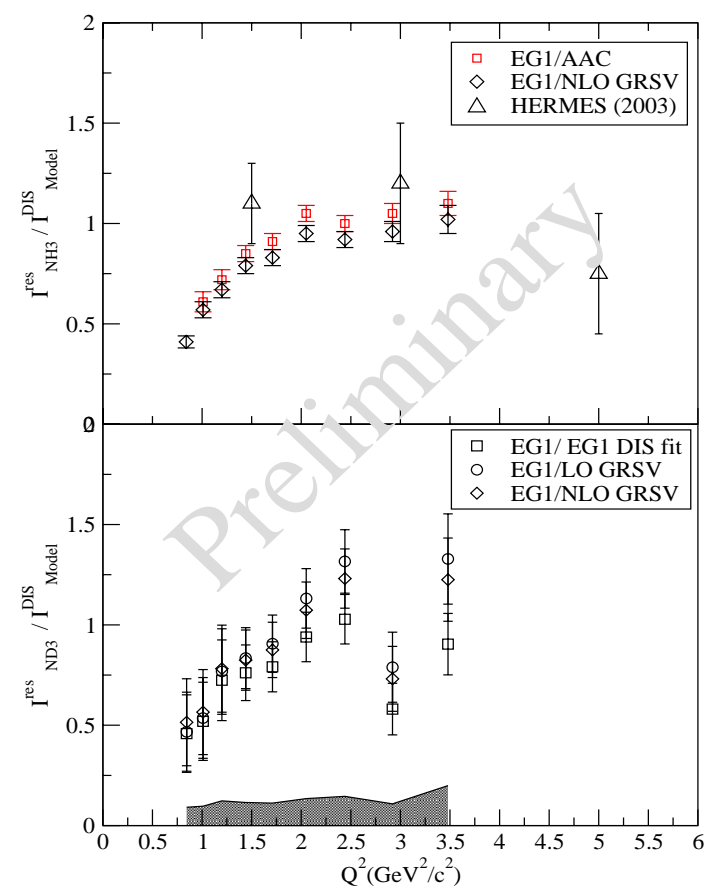

Figure 4. $\quad \Gamma_{1}^{R E S}\left(Q^{2}\right) / \Gamma_{1}^{D I S}$ as a function of $Q^{2}$ measured by CLAS. The top plot shows the results for the proton while the bottom plot shows the results for the deuteron. The Hermes data are shown for comparison.

agreement with the HERMES data, but provide a much more precise and detailed picture of the onset of duality. The CLAS ratio test begins to fail at the two sigma level when $Q^{2}$ becomes less than $1 \mathrm{GeV}^{2}$. The ratio is also consistent with unity at the $10 \%$ level for the proton and $30 \%$ level for the deuteron when $Q^{2}$ goes beyond $2 \mathrm{GeV}^{2}$.

\section{Higher Twist Contribution to Spin Structure Function}

Understanding the origin and quantify higher twist contributions to the structure functions is one of the key issues in the phenomenology of nucleon structure in the soft regime. In the case of spin structure functions, an interesting feature of $g_{2}\left(x, Q^{2}\right)$ is that it contains twist- 3 contributions at leading order. As a result, the integral $d_{2}=\int x^{2}\left(2 g_{1}\left(x, Q^{2}\right)+3 g_{2}\left(x, Q^{2}\right)\right) d x$ gives a measure of the higher twist contribution to $g_{2}$. The lower panel in Figure 2 shows the Hall A measurement of $d_{2}$ on the neutron for $Q^{2}=0.2-0.9 \mathrm{GeV}^{2}$. The data are in good agreement with the prediction of the MAID model ${ }^{32}$, 
while $\chi \mathrm{PT}$ calculations overestimate the integral even at the smaller $Q^{2}$. At $Q^{2}=5 \mathrm{GeV}^{2}$ the SLAC data give a large and positive value for the integral while lattice QCD calculations give a small and negative value, in better agreement with the trend of the present measurement.

\section{Summary and Outlook}

Extensive measurements of spin observables in the soft regime have been performed at Jefferson Lab involving the three experimental Halls. These measurements aim at understanding the transition region between hadronic and partonic degrees of freedom, investigating phenomena such as resonance excitation, duality in spin structure functions, higher twist contribution. The high precision results obtained from such experiments provide stringent constraint for theories in a kinematic region that was not explored in detail in the past.

In addition to the already completed measurements, new experiments are presently under analysis or planned for the near future. These include an extension of the Hall A and Hall B measurement of spin structure functions at very low $Q^{2}{ }^{9,35}$ with the goal of reaching a minimum $Q^{2}$ of 0.01 $\mathrm{GeV}^{2}$ in the measurement of $\Gamma_{1}$ to study the behavior of the integral at very low $Q^{2}$ and test chiral perturbation theory predictions. Furthermore, extensions of the duality studies on spin structure functions are planned in Hall $\mathrm{A}$ and $\mathrm{C}$ with experiment E01-012 ${ }^{11}$ which is presently under analysis and experiment E03-106 ${ }^{36}$ which will take data in the near future.

The already available data as well as the new data provided by the upcoming experiments will provide high precision information on spin observables and will hopefully allow us to reach a complete understanding of the nucleon spin structure in the soft regime.

\section{Acknowledgments}

I am grateful to the many members of the Hall A, CLAS, and Hall C Collaborations whose work I have reported here. The Southeastern Universities Research Association (SURA) operates the Thomas Jefferson National Accelerator Facility for the United States Department of Energy under contract DE-AC05-84ER40150.

\section{References}

1. For a recent review seeand references therein: B. W. Filippone and X. D. Ji, hep-ph/0101224 (2001). 
2. M. Garcon, these proceedings.

3. A. Deur, these proceedings.

4. P. Bosted, these proceedings.

5. M. D. Mestayer, these proceedings.

6. S. Frullani, J. Kelly, and A. Sarty, Jefferson Lab Proposal E91-011, (1991).

7. G. Cates, J. P. Chen and Z. E. Meziani, Jefferson Lab Proposal E94-010, (1994).

8. T. Averett and W. Korsch,Jefferson Lab Proposal E97-103, (1997).

9. J. P. Chen, A. Deur and F. Garibaldi, Jefferson Lab Proposal E97-110, (1997).

10. J. P. Chen, Z. E.Meziani and P. Souder,E99-117, (1999).

11. J. P. Chen, S. Choi, and N. Liyanage, Jefferson Lab Proposal E01-012, (2001).

12. J. Alcorn et al., Nucl. Instrum. and Meth. A522, 171 (2004).

13. B. Mecking et al. (CLAS Collaboration), Nucl. Instrum. and Meth. A503 (2003) 513.

14. V. D. Burkert, D. G. Crabb and R. Minehart, Jefferson Lab Proposal E91023, (1991).

15. S. E. Kuhn, G. E. Dodge and M. Taiuti, Jefferson Lab Proposal E93-009, (1993).

16. M. Anghinolfi, R. Minehart and H. Weller, Jefferson Lab Proposal E93-036, (1993).

17. P. Stoler, R. Minehart and M. Taiuti, Jefferson Lab Proposal E94-003, (1994).

18. C. D. Keith et al.: Nucl. Instrum. and Meth. A501 (2003) 327.

19. JLab E01-006, Spokesperson O. Rondon-Aramayo.

20. D. G. Crabb et al., Nucl. Instrum. and Meth. A356, 9 (1995); T. Averett et al., Nucl. Instrum. Meth. A427, 440 (1999).

21. J. D. Bjorken, Phys. Rev. 148, 1467 (1966).

22. H. Burkhardt and W. N. Cottingham, Annals of Physics 56, 453 (1970).

23. S. B. Gerasimov, Sov. J. Nucl. Phys. 2, 430 (1966).

24. S. D. Drell and A. C. Hearn, Phys. Rev. Lett. 16, 908 (1966).

25. V. Bernard et al., hep-ph/0203167 (2002).

26. X. D. Ji et al., Phys. Lett. B472, 1 (2000).

27. V. D. Burkert and B. L. Ioffe, Phys. Lett. B296, 223 (1992); J. Exp. Theor. Phys. 78, 619 (1994).

28. J. Soffer and O. V. Teryaev, Phys. Rev. Lett. 70, 3372 (1993), Phys. Rev. D51, 25 (1995), Phys. Lett. B 545, 232 (2002).

29. M. Amarian al., Phys. Rev. Lett. 92, 022301 (2004).

30. A. Deur et a., Phys. Rev. Lett. 93, 212001 (2004).

31. E. Thomas and N. Bianchi, Nucl. Phys. B82, 256 (2000).

32. D. Drechsel et al., Nucl. Phys A645, 145 (1999).

33. E. D. Bloom and F. J. Gilman, Phys. Rev. Lett. 25, 1140 (1970).

34. A. Airapetian et al.(HERMES Collaboration), Phys. Rev. Lett.. 90, 092002 (2003).

35. M. Battaglieri, A. Deur, R. De Vita, and M. Ripani, The GDH Sum Rule with Nearly Real Photons and the $g_{1}$ Structure Function of the Proton at Very Low $Q^{2}$, Jefferson Lab Proposal E03-006, (2003).

36. M. Jones et. al, Jefferson Lab Proposal E03-106, (2003). 